\title{
LIBÉRALISATION ÉCONOMIQUE ET CRISE POLITIQUE EN CÔTE D'IVOIRE
}

Bruno Losch

Presses de Sciences Po (P.F.N.S.P.) | «Critique internationale »

2003/2 n ${ }^{0} 19 \mid$ pages 48 à 60

ISSN 1290-7839

ISBN 2724629701

Article disponible en ligne à l'adresse :

http://www.cairn.info/revue-critique-internationale-2003-2-page-48.htm

\section{Pour citer cet article :}

Bruno Losch, «Libéralisation économique et crise politique en Côte d'Ivoire », Critique internationale 2003/2 ( $\left.\mathrm{n}^{\circ} 19\right)$, p. 48-60.

DOI 10.3917/crii.019.0048

Distribution électronique Cairn.info pour Presses de Sciences Po (P.F.N.S.P.).

(C) Presses de Sciences Po (P.F.N.S.P.). Tous droits réservés pour tous pays.

La reproduction ou représentation de cet article, notamment par photocopie, n'est autorisée que dans les limites des conditions générales d'utilisation du site ou, le cas échéant, des conditions générales de la licence souscrite par votre établissement. Toute autre reproduction ou représentation, en tout ou partie, sous quelque forme et de quelque manière que ce soit, est interdite sauf accord préalable et écrit de l'éditeur, en dehors des cas prévus par la législation en vigueur en France. Il est précisé que son stockage dans une base de données est également interdit. 


\title{
Libéralisation
}

Contre-jour économique et crise politique en Côte d'Ivoire

\author{
Entretien avec Bruno Losch, \\ économiste au CIRAD
}

ouvez-vous nous restituer les principales orientations de la réforme de libéralisation du secteur agricole au cours des années 1990 en vous centrant sur la filière cacao ? Avant de parler de la libéralisation, il est important de rappeler l'organisation antérieure du dispositif de gestion de la filière cacao. Âgé de près de cinquante ans, celuici avait été mis en place avant l'indépendance ; il articulait une caisse de stabilisation, qui gérait l'offre nationale ivoirienne, et des opérateurs privés, à l'origine essentiellement étrangers, mais qui se sont progressivement « ivoirisés ». La commercialisation intérieure était entièrement administrée par l'État, qui fixait les prix, et les exportations étaient soumises à des quotas définis par la Caisse - c'est-à-dire par le pouvoir politique - qui permettaient de répartir la manne cacaoyère entre les différents acteurs économiques, eux-mêmes parties prenantes du jeu politique. La grande particularité du système ivoirien tenait à cet emboîtement complet de l'économique et du politique avec, en son centre, ce que j'ai appelé le « complexe cafécacao $\gg^{1}$. L'alliance passée peu avant l'indépendance entre Houphouët-Boigny, père de la Nation et président omnipotent, et des entrepreneurs privés d'origine étrangère a reposé sur un arrangement : l'octroi aux firmes de certains avantages, notamment fiscaux, en échange de leur « compréhension » à l'égard de la cooptation, à leurs côtés, de personnalités ivoiriennes (d'abord par le biais des conseils d'administration puis par l'entrée progressive dans l'actionnariat des sociétés, notamment celles d'exportation du café et du cacao). Au milieu des années 1980, juste avant que la Côte d'Ivoire ne soit emportée par la crise de la dette et la chute des marchés internationaux, contrainte à l'ajustement structurel et à la libéralisation, ce système était donc totalement verrouillé et le pouvoir politique pouvait jouer sur les quotas d'exportation pour favoriser l'un ou l'autre des acteurs de la place.

Dès le début des réformes, les bailleurs de fonds ont eu dans leur ligne de mire les jeux de pouvoir et les transactions financières autour de la manne café-cacao. Lors de la première phase, celle de l'ajustement structurel stricto sensu et de l'assai- 
nissement financier, les privatisations et le désengagement de l'État n'ont pas dépassé le stade de l'incantation, car le rapport de forces entre le national et l'international, et la posture d'Houphouët, permettaient de résister et d'opérer des contournements permanents : par exemple en changeant le statut des entreprises (passage de sociétés d'État à des sociétés d'économie mixte) ou en supprimant certaines d'entre elles pour en créer immédiatement d'autres. L'épisode catastrophique de la « guerre du cacao $»^{2}$, à la fin des années 1980, mit le pays financièrement à genoux et changea le rapport de forces : le gouvernement Ouattara eut pour mission de passer au cran supérieur dans le processus de libéralisation, et notamment de s'attaquer, enfin, à la filière cacao. Les années 1990 seront celles d'une lutte pied à pied du pouvoir politique et des opérateurs privés ivoiriens pour résister au démantèlement de la Caisse. Les choses se font petit à petit : on a d'abord la remise en cause des quotas d'exportation, puis la libéralisation progressive de la commercialisation intérieure (suppression du barème des prix puis du prix officiel garanti aux producteurs) et, enfin, le rétrécissement des interventions de la Caisse à l'exportation, ses autorisations de vente étant remplacées par un pseudo système d'enchères. À chaque fois que l'État bénéficiera d'un ballon d'oxygène (dévaluation, reprise des cours), il y aura des retours en arrière. Mais, en 1999, la suppression de la Caisse de stabilisation est inéluctable.

Ce bouleversement du jeu intérieur s'est trouvé démultiplié par la recomposition simultanée du marché international du cacao, avec des synergies et des interférences permanentes entre le jeu intérieur et le jeu international : on assiste ainsi à la lutte acharnée des Ivoiriens pour tenter de conserver les rentes antérieures, mais aussi à l'élaboration d'une stratégie offensive, avec le renforcement du secteur industriel (du broyage de cacao) et de l'intégration verticale, grâce à l'alliance avec de grandes firmes étrangères, qu'illustre parfaitement la trajectoire d'une société comme SIFCA qui devient le fleuron du secteur.

Néanmoins, après avoir épuisé toutes les astuces pour freiner le processus, la libéralisation de la filière cacao est totale en août 1999, venant après celle du café en 1997. Et... clin d'œil de l'histoire, le coup d'État survient au mois de décembre de la même année.

Justement, ce qui est frappant, ce sont ces deux chronologies. Quelle articulation faitesvous entre la chronologie économique, avec le processus de libéralisation qui s'étend sur plus d'une dizaine d'années, marquant l'essoufflement de l'économie rentière d'origine coloniale dès la fin des années 1970, et la chronologie politique, avec le coup d'État de 1999 sanctionnant un long processus de pourrissement, d'exacerbation des luttes factionnelles, et de tensions agraires dans le Sud-Ouest ?

On ne peut pas dire, bien évidemment, que la fin de la Caisse explique le coup d'État. $\mathrm{Non}^{3}$. Il faut faire une relecture des années 1990 à la lumière des choix des décennies antérieures. Pour moi, ce qui est déterminant dans l'histoire économique et 
politique de la Côte d'Ivoire indépendante, ce sont justement les options qui ont été retenues avant, dans les années 1950, et qui sont remises en cause dans les années 1980. Ce qui débouche sur ce que j'appelle le «double déclassement » de la Côte d'Ivoire.

Déclassement économique, tout d'abord, avec l'épuisement de la stratégie économique initiale, caractérisée, on l'a vu, par l'alliance avec les firmes étrangères mais aussi par la poursuite des choix coloniaux, c'est-à-dire la spécialisation dans l'exportation de produits agricoles de base, qui retire toute marge de manœuvre au pays. Ce positionnement a été longtemps payant puisqu'il a bénéficié de près de cinquante ans de protection relative, grâce aux garanties de rémunération qu'ont offertes successivement le cadre douanier colonial (dès les années 1930), puis les accords internationaux sur les produits de base ou encore les privilèges obtenus de la Communauté européenne.

Mais, à partir des années 1980, à la suite des programmes de libéralisation et du désengagement des États, les caisses de stabilisation et les offices de commercialisation sont partout démantelés. Parallèlement, les firmes du négoce et de l'industrie entament des restructurations de grande ampleur qui donnent naissance à des « géants » mondiaux. L'oligopole d'États-nations est remplacé par un oligopole de firmes. Et la Côte d'Ivoire et sa Caisse se retrouvent face à des acteurs d'une autre nature. Engluée dans son complexe café-cacao, otage des compromis économicopolitiques rentiers et paralysée par un pouvoir présidentiel vieillissant, la Côte d'Ivoire perd des occasions de s'adapter à la nouvelle donne. La « guerre du cacao », de 1987 à 1989 (avec l'arrêt des exportations par Houphouët-Boigny), en est un symbole : elle a été perdue à cause de la sous-estimation du nouveau rapport de forces.

Le second déclassement est géopolitique. La Côte d'Ivoire avait bénéficié, du fait de ses options libérales et pro-occidentales et de son rôle de vitrine et de relais de la France au sud du Sahara, d'un statut privilégié. La fin des années 1980 sonne aussi, avec la fin de la guerre froide, la remise en cause de cet ordre géopolitique antérieur dans lequel la Côte d'Ivoire avait été maintenue dans une bulle (économique, financière, institutionnelle, politique). Mais il y avait aussi la stature d'Houphouët : il suffit de rappeler que la dévaluation du franc CFA, qui symbolise ce déclassement, n'a eu lieu qu'après le décès du «Vieux », qui lui était fondamentalement opposé.

La conséquence de ce double déclassement est l'érosion des marges de manœuvre du pouvoir politique, la perte de ses différents leviers de régulation économique et sociale. Elle se traduit par la remise en cause des compromis fondateurs de l'État national ivoirien entre le pouvoir politique et, d'une part les planteurs, de l'autre les entreprises étrangères. Ce double compromis comptait des arrangements connexes qui, eux aussi, ont été malmenés : avec les travailleurs migrants (possibilités d'accès à la terre, aux soins, au vote), avec les autochtones du Sud 
(acceptation des migrants, accès au foncier facilité par les perspectives d'ascension sociale, par l'école et l'emploi salarié, dans la fonction publique et le secteur privé), avec une petite bourgeoisie émergente issue de la fonction publique (qui bénéficiait d'avantages divers, tels que des indemnités et des logements).

La réduction des marges de manœuvre dans les années 1980, qui s'aggrave drastiquement au début des années 1990, va se traduire par des ruptures et une série de revendications : des revendications catégorielles, liées à la suppression des avantages acquis par les enseignants, les médecins, les fonctionnaires en général et les « corps habillés » (militaires, douaniers...) ; mais aussi des revendications à caractère régional consécutives à l'assèchement des politiques de rééquilibrage ; et, enfin, des revendications politiques marquées par la contestation, au sein du parti unique, des jeunes générations face à un pouvoir présidentiel vieillissant. Tout cela cause le mécontentement d'une grande partie de la population : les «gens du Nord » se sentent délaissés, les « autochtones du Sud » ont le sentiment d'être les dupes d'un projet d'ascension sociale qui ne fonctionne plus. Il faut bien garder à l'esprit que le revenu moyen par tête est divisé par plus de deux entre 1980 et 2000 !

Donc, les années 1990 inversent ces processus et érodent les mécanismes permettant les compromis sociopolitiques. Quelles stratégies les Ivoiriens vont-ils alors déployer face à cette crise multiforme ? Comment les opérateurs nationaux, notamment, qui avaient engagé une stratégie d'industrialisation et d'intégration, réagissent-ils ?

La libéralisation progressive de la filière cacao et la remise en cause des quotas d'exportation font disparaître ce que l'on appelait les « quotataires politiques », poussant à une concentration au profit des groupes industriels déjà installés, des étrangers mais aussi de quelques Ivoiriens qui se sont imposés dans le jeu économique local. Ces recompositions sont accélérées par les privatisations, qui donnent des opportunités sans précédent aux firmes nationales proches du pouvoir, les mieux placées pour se partager les dépouilles de l'État. Mais ces firmes nationales font aussi le choix, contraint par le nouveau rapport de forces international, des alliances avec les grandes firmes étrangères. En effet, à partir du moment où l'accès à la rente cacaoyère n'est plus garanti par les quotas de la Caisse, où le système de caution et de garantie du financement de la commercialisation par l'État n'existe plus, les firmes ivoiriennes ont un besoin crucial de liquidités, faute de pouvoir s'appuyer sur un système bancaire solide et prêt à prendre des risques. Inversement, les firmes étrangères (broyeurs et négociants), qui n'ont plus de garantie d'accès à la production ivoirienne en raison de la disparition des quotas et du démantèlement progressif de la Caisse, ont - elles aussi - intérêt à s'allier avec des firmes locales. Cet intérêt réciproque repose sur l'échange de l'accès aux financements internationaux (grâce à l'adossement aux grands groupes mondiaux) en contrepartie de l'accès au cacao ivoirien qui, il faut le rappeler, constitue désormais $45 \%$ de l'offre mondiale. Parallèlement, les firmes ivoiriennes élaborent une véritable stratégie 
industrielle pour gagner des marges de manœuvre sur des marchés internationaux de plus en plus compétitifs, s'appuyant sur un effort d'intégration verticale et de réponse plus adaptée aux nouvelles exigences de qualité des chocolatiers. On assiste, d'une part, à une stratégie de développement du broyage en Côte d'Ivoire (il s'agit d'exporter non pas des fèves mais du beurre de cacao) et, d'autre part, à une stratégie de développement en aval visant à bénéficier d'un meilleur positionnement sur le marché. L'entreprise la plus performante à cet égard est SIFCA, qui se structure en holding - SIFCOM - à l'occasion des privatisations et devient un groupe complètement ivoirien au tournant des années 1990. Fondée en 19571958 par des capitaux français (Tardivat), SIFCA a été progressivement grignotée de l'intérieur par des capitaux ivoiriens, au terme d'un jeu complexe d'actionnariat croisé et de manœuvres politiques. En 1992, elle passe ainsi entièrement sous contrôle local, avec Bédié (qui n'est pas encore président) et quelques ténors politiques comme principaux actionnaires. SIFCA investit alors en aval, créant une unité de production de beurre de cacao liquide en France, à Dunkerque (Nord Cacao), et rachetant une fabrique de poudre de cacao espagnole (Incresa). On a là une stratégie industrielle effective, exceptionnelle en Afrique, de la part d'une entreprise devenue totalement ivoirienne, qui va jusqu'à des investissements en Europe. SIFCOM profite simultanément de sa position économico-politique particulière (surtout après l'accession de Bédié au pouvoir) pour « rafler » de nombreuses opportunités offertes par la privatisation de l'économie ivoirienne, dont elle rachète plusieurs fleurons agro-industriels, ou pour récupérer les dépouilles de certains de ses concurrents locaux comme JAG, deuxième exportateur ivoirien, détenu par l'un des golden boys des années 1990, François Bakou. Le groupe se constitue alors un empire conséquent, au point qu'en 1999 SIFCA était devenue le premier groupe agro-industriel africain (hors Afrique du Sud). SIFCOM assure aussi sa croissance par la diversification (à travers sa filiale Comafrique) vers d'autres secteurs, comme l'import-export, la pharmacie ou le rachat d'entreprises privatisées, notamment les télécommunications ivoiriennes en partenariat avec France Telecom.

Pour revenir à votre question initiale, on peut dire que la disparition annoncée de la Caisse, en signifiant la perte, pour le pouvoir politique, de la possibilité de faire de « gros coups » (par les quotas et le déblocage des ventes aux favoris du régime), a conduit la classe politique à se repositionner, de l'appareil d'État vers le nouveau secteur privé libéralisé. Jusqu’à sa fin, la Caisse interviendra dans le jeu politique local, mais désormais en synergie avec les grosses firmes ivoiriennes qui contribuent au financement des campagnes électorales, ainsi que des mouvements bédiéistes comme Le Jardin Réveil.

On se trouve donc, avec SIFCA-SIFCOM, en présence d'un Goliath, premier exportateur de cacao du monde. Mais un Goliath aux pieds d'argile car, en termes de capacité de trésorerie, le groupe dépend de financements extérieurs qui sont faci- 
lités par ses alliances externes, notamment avec le broyeur hollandais De Zaan contrôlé par l'américain Grace Cocoa. On pourrait expliquer l'effondrement du capitalisme industriel ivoirien par l'absence de capitalisme bancaire local. SIFCA bute tout simplement sur des problèmes de financement. Tant que Bédié, en tant qu'actionnaire, et la Caisse servaient de caution sur les marchés internationaux, tout allait bien. Mais, avec la disparition de la Caisse et, surtout, avec le coup d'État de décembre 1999, SIFCA perd ses appuis structurels. Dès 2000, SIFCA, qui a par ailleurs opéré de mauvais positionnements sur le marché, se trouve en porte-à-faux, d'autant plus que le jeu des stratégies industrielles mondiales l'ont entre-temps desservie. Grace Cocoa, qui avait renforcé sa participation au tour de table en 1996, est en effet passé à la même époque dans l'escarcelle d'une autre société américaine $(\mathrm{ADM})$, devenue rapidement le premier broyeur mondial. Pour sortir de son étranglement financier, SIFCA doit alors se désengager et vendre ses actifs industriels dans le cacao. Et c'est ADM, principal actionnaire minoritaire, qui rafle la mise. La plupart des autres grandes firmes ivoiriennes ont subi un sort identique : surendettement et rachat. Aujourd'hui, les trois plus grands broyeurs mondiaux sont installés en Côte d'Ivoire et contrôlent les exportations ivoiriennes.

Il y a donc remplacement du régime néo-mercantiliste, au centre duquel on trouvait la Caisse de stabilisation, par un oligopole privé étranger. Quel est le lien entre le déclin du capitalisme ivoirien et l'exacerbation du nationalisme économique qui s'exprime à travers des attaques contre Bouygues ou Bolloré, et va de pair avec un certain nationalisme politique qu'incarnent un courant du parti de Gbagbo et les " jeunes patriotes " ?

Curieusement, ces réactions nationalistes s'expriment surtout vis-à-vis des firmes françaises, alors même que les grands exportateurs de cacao sont américains ou européens (franco-alémano-suisse dans le cas de Barry-Callebaut). La prise de conscience, dans le secteur cacaoyer, du pouvoir considérable de ces grands exportateurs s'est traduite par la volonté des autorités politiques de mettre en place des institutions de « gouvernance » privée pour tenter de leur faire contrepoids. Mais, paradoxalement, ce ne sont jamais ces firmes qui sont montrées du doigt quand on veut mettre en avant la domination étrangère : ce sont systématiquement les entreprises françaises, et principalement Bouygues et Bolloré. Je manque d'éléments pour affirmer que cette dénonciation s'explique par le fait que ces deux entreprises ont bénéficié de privatisations quand Ouattara était Premier ministre. Mais le constat est troublant. À l'époque, déjà, une grande campagne avait été orchestrée contre la libéralisation à outrance (instrumentalisée par Bédié) et la privatisation au pas de charge de l'eau et de l'électricité au profit de Bouygues. Cette entreprise était alors devenue l'épouvantail, symbole à la fois d'une perte de contrôle national sur les services de base et d'un bradage par quelqu'un de clairement identifié sur l'échiquier politique. De même, Bolloré avait largement accru son contrôle dans le domaine des transports, puis dans les plantations industrielles. Ce sont les 
premières privatisations, très visibles ; on en a beaucoup parlé, car c'était un phénomène nouveau en Côte d'Ivoire à l'époque. Les recompositions du secteur du cacao, en revanche, ont été d'une telle complexité qu'elles ont été plus difficiles à saisir. La presse locale n'a jamais sérieusement investi ce sujet. En outre, dans les années 1990, tout ce qui s'est joué autour de Bakou et de SIFCA était tellement politique que, finalement, on en parlait relativement peu. Il était très facile pour Bédié de faire parler sur Bouygues, car « Bouygues, c'était Ouattara ». La crispation nationaliste utilise aujourd'hui le bradage de l'économie nationale, mais, curieusement, on ne cite pas le secteur stratégique de l'économie ivoirienne : celui du cacao.

\section{Comment cet oligopole d'opérateurs privés fonctionne-t-il aujourd'hui en liaison avec les} opérateurs politiques?

Pour être honnête, il m'est difficile de répondre étant donné l'extrême instabilité dans laquelle on est depuis 2000. Il y a d'abord eu une période de transition, pendant laquelle les réformes institutionnelles se sont théoriquement mises en place. Du fait du caractère stratégique de la filière, les Ivoiriens ont réussi à obtenir des bailleurs de fonds la mise en place d'un dispositif de « gouvernance » privé, avec la création de plusieurs institutions : une autorité de régulation, société d'État qui ne dispose d'aucun pouvoir d'intervention direct mais vérifie le respect des règles de concurrence et supervise les agréments des exportateurs; une « bourse du cafécacao » qui est en réalité une autorité de régulation des exportations ivoiriennes ; un fonds de régulation et de contrôle censé mettre en place un système de stabilisation intra-annuel qui permette d'offrir un prix de campagne indicatif et de sécuriser l'environnement des producteurs ; un fonds de promotion de développement de la caféiculture et de la cacaoculture ; et, enfin, un fonds de garantie pour les coopératives. Tous ces nouveaux organismes, créés dans des conditions politiques difficiles, ont une gestion privée, avec un conseil d'administration.

\section{Justement, par qui sont nommés ces conseils d'administration ?}

On est, là, dans le clair-obscur le plus total ! Il y a bien quelques représentants de l'État, mais les membres sont dans l'ensemble nommés par les opérateurs privés. Dans chacune de ces instances, on trouve des représentants des banques, des exportateurs et des « planteurs ». Ce qui pose bien sûr la question du « comment on représente les planteurs »! Au terme d'un jeu subtil, l'année 2000-2001 a vu l'apparition providentielle de l'ANAPROCI (Association nationale des producteurs de café-cacao de Côte d'Ivoire, dont les membres ont été, de fait, cooptés par l'État sur une base régionale), auto-promue par le ministère de l'Agriculture comme l'interlocutrice représentative des planteurs. C'est donc l'ANAPROCI qui a désigné des représentants dans les conseils d'administration, souvent (mais non exclusivement) d'anciens fonctionnaires reconvertis : bel exemple de l'adaptation de la « société civile » à la libéralisation ! 


\section{Qui a le pouvoir dans ces organismes ? Y retrouve-t-on la domination des intérêts étrangers ?}

Non. Dans tous ces organismes, ce sont toujours les « planteurs » qui ont le pouvoir. Les autres sont minoritaires : les banques et les exportateurs, autrement dit les grandes firmes internationales, sont plutôt en position de devoir subir, en soupirant, les turpitudes et ce qu'ils doivent considérer comme du folklore local. Ils sont un peu les otages de ces institutions qui marchent très mal. Tous ces organismes sont alimentés par des prélèvements autoritaires sur la filière : en plus des droits d'enregistrement et de sortie, qui restent très importants (220 FCFA/kg), une contribution automatique est versée aux différents fonds gérés officiellement par ces conseils d'administration, donc de façon totalement privée. Les sommes en jeu sont « significatives » : sur la campagne 2001-2002 et les trois premiers mois de la campagne suivante, l'ensemble de ces ponctions s'est élevé à 580 milliards de francs CFA (884 millions d'euros), dont 400 pour l'État et 180 pour ces organismes de gestion, soit un montant équivalent à l'ensemble des concours extérieurs attendus par le gouvernement dans le budget 2002 ! On est dans un clair-obscur... plutôt obscur, car tout cela se passe dans un contexte politique d'une mouvance totale. Ces organismes fonctionnent très mal : ils n'ont pas de comptes, pas de budget. On ne sait pas grand-chose sur eux, mais ce dont on est certain, c'est que le prix des jetons de présence aux conseils d'administration est élevé : 500000 francs CFA par séance et 800000 francs CFA hors Abidjan... On comprend que la plupart des réunions aient lieu à Bassam ! S'il y a vraiment des planteurs d'origine, en quelques séances, ils ont complètement changé d'univers ! Autre exemple : le fonds de régulation et de contrôle n'a jamais vraiment été mis en place ; mais, comme on voulait tout de même prélever pour la stabilisation, une réserve prudentielle, avec un compte séquestre ouvert à la $\mathrm{BCEAO}$, a été instituée pour recevoir les prélèvements. Or, il a été révélé que des transferts de ce compte-séquestre vers la Caisse autonome d'amortissement lui avaient notamment permis de rembourser une partie de la dette extérieure de la Côte d'Ivoire. Sans vouloir être médisant, on peut sans doute trouver là l'explication du mutisme de certains bailleurs de fonds face aux malversations évidentes et à l'échec de la gouvernance privée.

\section{Donc le syphonage continue, mais désormais sur un mode privé...}

Privé et beaucoup plus anarchique, car cela part dans tous les sens. Le Fonds de promotion de la caféiculture et de la cacaoculture prélève 35 francs CFA/ $\mathrm{kg}$ sur 1250000 tonnes annuelles, ce qui représente des sommes considérables. Ce pactole tout à fait significatif a servi à beaucoup de choses. Par exemple, les membres du conseil d'administration de l'ANAPROCI ont décidé de créer une société, l'ANAPROCI-SA ! Ce n'est pas l'association qui en est actionnaire, mais les membres de son conseil d'administration en tant que personnes physiques. Or, ANAPROCI-SA a bénéficié d'une dotation du Fonds de promotion pour racheter, sans intérêt commercial ou industriel, certains actifs de... SIFCA qui 
n'avaient pas été repris par ADM. Pour cette opération, une nouvelle société a vu le jour (SIFCA-Coop), qui a racheté des usines dont personne ne voulait. Ce qui permet en fait de redonner de la trésorerie et de la marge de manœuvre aux actionnaires historiques de SIFCOM. Il y a donc bien toujours une utilisation de la manne cacaoyère, mais les jeux sont encore plus opaques qu'auparavant, lorsqu'on se trouvait dans un univers relativement bordé. En référence à l'alliance braudélienne du prince, du marchand et du malfrat à la base de l'émergence du capitalisme, on peut dire qu'aujourd'hui, en Côte d'Ivoire, le prince est bien affaibli alors que le malfrat prospère !

Est-ce que le prince, ou ce qui en tient lieu, c'est-à-dire Laurent Gbagbo, est arrivé à prendre pied dans la filière ? Et, question subsidiaire, Bédié est-il totalement sorti de ce qui reste de I'empire SIFCOM ?

Certes, SIFCOM a perdu ses fleurons (SIFCA et Unicao), mais tout le reste est là, et les actionnaires aussi. Il est très difficile d'obtenir des informations de ce genre, mais je n'ai eu aucun écho selon lequel Bédié aurait été expulsé du tour de table. Il n'y a aucune raison à cela.

Mais peut-on voir l'ANAPROCI comme une émanation du pouvoir de Gbagbo?

Non, c'est beaucoup plus complexe et chaotique. Il y a certes eu une volonté du pouvoir politique, qui a pris conscience que, avec la disparition de la Caisse, la filière stratégique passait complètement sous contrôle étranger.

Qui en " a pris conscience ", concrètement ?

Plus que la tête du pouvoir politique, je pense que c'est la superstructure - c'est-àdire des responsables des ministères, des directeurs techniques, ainsi que les patrons de PME exportatrices liées à ces fonctionnaires. Ils se sont dit qu'il fallait absolument un contre-pouvoir à ces gros opérateurs privés étrangers. Or, le contrepouvoir, ce sont des organisations professionnelles et des structures de « gouvernance » privée qui permettent d'agir. La Côte d'Ivoire paie la facture de ses choix précédents et de sa négligence permanente envers la représentation professionnelle. On peut même aller plus loin : Houphouët a toujours tout fait pour qu'il n'y ait aucune représentation professionnelle. Et, pendant les dix années de libéralisation, rien n'a été entrepris en la matière. Quand tout s'est effondré, en 2000, on s'est retrouvé, avec les trois géants mondiaux du broyage, face à des organisations professionnelles qui n'en ont que le nom. La volonté de la superstructure s'est donc traduite par la création de cette ANAPROCI, montée de toutes pièces et dotée de tous les pouvoirs. De son côté, l'ANAPROCI joue un jeu autonome ; elle n'a pas de relation directe avec le pouvoir politique actuel. D'autant que Gbagbo est totalement étranger à cette histoire et à ses intrigues. Gbagbo est « tout neuf » dans cet univers.

Mais il y a un paradoxe, il vient tout de même d'une région cacaoyère ?

Oui, mais il a pleinement bénéficié du compromis historique et du système Houphouët. Il est issu de ce milieu qui, au lieu d'investir dans l'agriculture, a clai- 
rement opté pour la scolarisation, et son ascension sociale a été fulgurante : il est devenu professeur d'Université et président de la République ! Mais, du coup, il est étranger au jeu d'intrigues qui s'est tissé lentement, sur une quarantaine d'années, en Côte d'Ivoire : c'est l'outsider qui gagne par militantisme et par l'incapacité de ceux qui étaient au cœur du complexe à se repositionner dans ce contexte de déclassement et de recompositions internationales. Gbagbo a driblé tout le monde en 2000. Personne ne pariait sur lui au moment du coup d'État de 1999.

On aurait donc un FPI populiste, nationaliste, enraciné dans le Sud-Ouest cacaoyer, mais qui ne serait pas un parti agraire ?

Oui, le FPI est essentiellement un parti de villes, même s'il s'est développé rapidement dans certaines zones rurales à la faveur de la crise, principalement dans les anciennes zones cacaoyères. Toutes les recompositions des deux dernières années se font avec de nouveaux protagonistes politiques qui n'ont pas la culture des intrigues cacaoyères, même si leur apprentissage est rapide. L'ANAPROCI, on l'a vu, est née de la volonté du ministère de l'Agriculture de créer des contre-pouvoirs et de la « gouvernance » privée qui soient contrôlés par les producteurs. En pratique, on a utilisé l'appareil d'État, c'est-à-dire que l'on a demandé aux préfets et aux coopératives de donner le nom de deux personnes par département, et le tour était joué. Il n'y a pas eu d'élections. Ses membres devaient, théoriquement, être renouvelés lors d'élections générales, mais celles-ci n'ont jamais eu lieu. L'un des principaux protagonistes m'a donné une image suggestive du processus : « Le gouvernement a frotté sur la lampe d'Aladin et en a fait sortir l'ANAPROCI. Mais, maintenant que l'ANAPROCI existe, il n'arrive pas à l'y faire rentrer. » Les membres de l'ANAPROCI, quelques vieux planteurs mais aussi des cadres reconvertis, jouent de fait un jeu autonome ; ce qui n'exclut pas, bien évidemment, des alliances et des relais. Par exemple, le Fonds de promotion a été utilisé pour soutenir l'effort de guerre : un chèque de 10 milliards de francs CFA a été remis en grande pompe devant la presse au président Gbagbo par son président, Henri Amouzou, ancien membre d'un syndicat d'opposition qui était lié au FPI. Là, effectivement, il y a une connexion. De même, l'absence de réaction de l'ANAPROCI dans l'histoire du transfert de la réserve prudentielle à partir du compte-séquestre à la BCEAO montre bien ses liens avec certains hauts dignitaires du régime. Mais la structure de contrôle n'est plus la même qu'auparavant ; il y a désormais des connexions d'opportunité, du bricolage - un bricolage qui rapporte.

Cela dit, la rente cacaoyère n'est-elle pas compromise par la guerre civile et par la crise, qui conduit à une baisse de la qualité ?

Pour l'instant, la « Côte d'Ivoire utile », celle du cacao, est peu affectée. Le cacao « sort » du pays et, du coup, la situation macro-économique, qui bénéficie de la hausse des cours induite par le conflit, est plutôt bonne - à court terme - pour un pays en guerre. Quant à la baisse de la qualité, il n’y a là rien de significatif et, à la 
limite, les principaux acheteurs s'en moquent. Actuellement, on assiste à une certaine normalisation des standards de qualité au niveau international. L'important, pour les broyeurs, est d'avoir une garantie d'approvisionnement qui leur assure une quantité homogène et une régularité dans les assemblages et la fourniture des chocolatiers. Et leur objectif est de ne pas payer plus cher que leurs concurrents. Le problème de la qualité est secondaire, sauf pour certains chocolatiers « haut de gamme » qui ont des filières d'approvisionnement spécifiques.

Mais la guerre civile traduit une exacerbation du conflit politique et social. Comment le conflit politique s'est-il articulé avec le conflit social ? La crise économique a entraîné la rurbanisation du Sud-Ouest et le retour des jeunes dans les villages, des jeunes qui ont alimenté ces tensions sociales...

Pour l'anecdote, certains ont essayé d'établir un lien direct entre la rébellion et le cacao en affirmant que les grandes firmes de cacao avaient financé les opérations de déstabilisation ; c'est l'affaire Amajaro. Je n'y crois guère, même si une opération spéculative comme celle-là a effectivement pu servir à mobiliser des fonds... En revanche, plus structurellement, je ne pense pas qu'il y ait de lien direct avec le déclenchement de la rébellion. Aujourd'hui, celle-ci est cantonnée dans le Nord et dans l'Ouest. Elle n'affecte pas la zone cacaoyère à proprement parler ; elle est concentrée dans la région de Man, qui est l'une des rares régions à dominante caféière, et aux abords de la frontière libérienne, où il y a certes du cacao, mais rien de stratégique par rapport aux grands volumes des récoltes ivoiriennes. Il y a bien eu une poussée de fièvre lorsque les rebelles ont pris Daloa, mais elle a été de courte durée. Le risque serait qu'ils prennent le port de San Pedro, qui écoule 40 \% de la production ivoirienne.

Il y a néanmoins un lien indirect. Car ce qui se passe aujourd'hui ne peut se comprendre qu'au regard des délitements successifs, de l'érosion des anciennes régulations politiques et de la rupture d'un certain nombre de compromis historiques. Notamment la disparition de certaines contreparties à caractère régional et catégoriel, par exemple dans l'armée. Les populations du Nord ont exprimé leur rasle-bol : elles sont, depuis la fin des années fastes et la disparition d'Houphouët, marginalisées par rapport à un activisme de Bédié directement impliqué dans l'affairisme cacaoyer et industriel. De même, les « corps habillés » ont vu leurs conditions de rémunération se dégrader. Là où il y a concomitance, c'est entre le déclassement international du pays, qui se traduit par une rupture des compromis essentiels, et l'essoufflement du modèle de croissance basé sur l'exportation de produits de base. L'économie de plantation s'est construite sur un processus d'exploitation extensive de la forêt, en allant toujours plus loin vers l'Ouest. Or, dans les régions les plus anciennes, le vieillissement des vergers nécessitait de replanter. La replantation est aléatoire, coûteuse, demande de la main-d'œuvre et se traduit par une période de perte de revenus extrêmement importante. Cet essoufflement du modèle a été 
plus sensible dans des zones comme le Centre-Ouest, où la stratégie « urbaine » qui avait été adoptée ne fonctionne plus, où les jeunes n'ont plus de perspectives d'ascension sociale alors que l'accès à la terre leur est désormais difficile. Les jeunes déscolarisés qui retournent au village n'ont aucune marge de manœuvre : les terres ont été attribuées à des « étrangers », les vieux planteurs ne veulent pas céder la place. Il y a un vrai problème de générations en Côte d'Ivoire. Il faudrait réfléchir à la question du transfert inter-générationnel du patrimoine, dans le secteur agricole et cacaoyer en particulier. Or, cette question n'a jamais été abordée. Ce blocage des jeunes générations est un terreau tout à fait favorable au développement de la problématique xénophobe.

La libéralisation ne s'est pas forcément traduite par des résultats négatifs, mais par un contexte d'instabilité qui alimente un sentiment d'insécurité aussi bien sur le plan politique (le délitement de l'État, le développement de l'impunité, une police qui fonctionne mal...) que sur le plan économique (on ne sait pas quel sera le prix du cacao demain, combien de tonnes vont être enlevées, et, de plus en plus, si les cargaisons ne vont pas être attaquées). Il y a une perte des repères. La population ivoirienne, et notamment les jeunes, a le sentiment d'être dans une impasse, de s'être fait duper par les politiques. Tout cela se traduit par un repli sur des logiques identitaires, c'est-à-dire par une défiance générale vis-à-vis du politique national et par un repli sur des logiques de proximité où, souvent, les rhétoriques d'exclusion servent de référentiel et d'argumentaire.

\section{Y a-t-il une différence régionale dans la gestion de ces tensions sociales ?}

Oui. Dans le Sud-Est, les instances de régulation sociale ont dans l'ensemble mieux fonctionné et encadré les populations, alors que le choc était comparable. Les chefferies akan sont de vraies chefferies, avec une structuration solide. Les Baoulé, poussés par Houphouët à la conquête de la forêt à cacao, n'ont pas pu aller en pays agni ; ils se sont installés là où c'était le plus facile, c'est-à-dire en pays bété, par exemple, où l'organisation traditionnelle n'était pas aussi structurée. En fait, Houphouët a poussé les Baoulé dans le ventre mou de la Côte d'Ivoire. La différence, aujourd'hui, tient au fait que, dans le grand pays agni, la structuration sociale est restée beaucoup plus cohérente et solide qu'ailleurs.

\section{Et que se passe-t-il en pays baoulé même ?}

C'est difficile à dire, car le pays baoulé est aujourd'hui essentiellement situé en zone rebelle. On manque de recul. Ce que l'on peut constater, toutefois, c'est qu'en moins de deux ans de nouvelle présidence, cette région, qui était intimement liée aux deux premiers présidents, s'est retrouvée en zone rebelle. Il est très probable que le problème des « étrangers » cache aujourd'hui la question baoulé. Tout est en place pour que cette question se pose aussi. 
Il y aurait en fait une double question baoulé, celle des planteurs migrants et celle du contrôle d'intérêts baoulé sur certains lambeaux de la filière cacao ?

Sur l'économie de plantation, cela ne fait aucun doute à terme. Sur les intrigues économico-politiques, je ne pense pas. Je dirais qu'en Côte d'Ivoire le capitalisme est davantage un capitalisme de grands commis de l'État, ou un capitalisme politique, qu'un capitalisme baoulé. Dans l'actionnariat des firmes, on trouve plutôt des individus qui s'inscrivent dans la logique de répartition de la rente entre les différentes catégories socio-professionnelles et régionales, que cela soit les quotataires politiques des années 1970 ou les actionnaires des années 1980-1990. Il n’y a pas de capitalisme baoulé stricto sensu, au sens ethnique du terme.

Propos recueillis le $1^{\text {er }}$ mars 2003 par Jean-François Bayart et Béatrice Hibou

Bruno Losch est économiste au CIRAD. II est l'auteur de nombreux travaux sur l'économie politique ivoirienne et a récemment coordonné une étude pour la DGCID du ministère français des Affaires étrangères sur l'impact de la libéralisation de l'économie ivoirienne.

E-mail : losch@cirad.fr

1. B. Losch, Le complexe café-cacao de la Côte d'Ivoire. Une relecture de la trajectoire ivoirienne, thèse d'économie, Université de Montpellier, 1999.

2. Pour un récit romancé mais néanmoins précis, voir J.-L. Gombeaud, C. Moutout et S. Smith, La guerre du cacao. Histoire secrète d'un embargo, Paris, Calmann-Lévy, 1990.

3. Voir sur ce point B. Losch, « Coup de cacao en Côte d'Ivoire. Économie politique d'une crise structurelle», Critique internationale, $\mathrm{n}^{\circ}$ 9, octobre 2000, pp. 6-14. 